\title{
Effective Model and Methods for Analysing Human Factors in Software Design for Efficient User Experience
}

Sami Abduljalil, Dae-Ki Kang (corresponding author), Member, KIMICS

\begin{abstract}
In software system development, an application interface is the main communication platform between human developers and applications. Interaction in any software application requires human's mental and physical activities. Although software systems have increased drastically in diverse sectors and many forms to quench human's needs and satisfactions, human always concern about the ease in usability of the software application so that it can be easily understood and navigated. Since many software developers still focus on the quantity of contents instead of the quality of the interface from the user's point of view, it is important to address human factors need in the early stage of the design and to continue addressing them during the entire stages of the software design for the persistent support of usability. In this paper, we propose the Modified Prototype Model (MPM), which helps the software designers and developers to design user-friendly software systems with easy-to-navigate interfaces by uncovering human factors in a convenient way. Moreover, we propose methods that assist to identify more human factors regarding software design. In this paper, we also study the implications of the proposed model and the proposed methods.
\end{abstract}

Index Terms- Human Factors, Software Design, Web Design, Usability.

\section{INTRODUCTION}

With amazingly quick and rapid application and software design in various forms, the need for studying human factors is emerged. In order to capture human factors entirely in any application or software design is obviously ongoing research across diverse aspects and sectors. In the last decades, the research of the usability was effectively and widely done by many researchers to improve the quality of the design $[1,2,3]$. Human computer interface is an intermediary in a communication between people and computers. In other words, human computer design (interface) is a platform of ongoing information flow and feedback between human and computers. Efficiency of the communication, information flow and feedback between people and computers

Manuscript received January 12, 2011; revised February 1, 2011; accepted February 7, 2011.

Sami Abduljalil is with the Ubiquitous IT Graduate School, Dongseo University, Pusan, 617-716, South Korea. (samialkindi0708@gmail.com)

Corresponding author Dae-Ki Kang is with the Division of Computer \& Information Engineering, Dongseo University, Pusan, 617-716, South Korea. (dkkang@dongseo.ac.kr) depends on the effectiveness of good structure, the readable contents and the motivated tasks in any software application [4].

The acknowledgement of the human needs and requirements in the design and deployment of quality and user-centered design can ensure the success of the interactions between human and application. A human factor is potentially playing a significant role in success of product quality and reliability, so poorly designed human computer interfaces can lead to many unexpected problems. Neglecting the importance of the human factors can leave a huge impact on the success of the products and cause people to quit or leave the intervention in the application or quitting using the products besides causing frustration to human.

Many computer interface designs have caused fatal damage to human and machines as well. One good example of human computer design errors that liable many people to death is Therac25. Therac25 is an accident that occurred in United State and Canada between June 1985 and January 1987. The Therac25 machines cause death to seven persons by overdosing the patients. Due to lack of coherent structure, readability and proper procedure in the system interface, the person pressed the wrong button which caused the accident to occur [5]. On the other hands, the goal of studying the human factors is to quickly utilize human needs and requirements as a main source in designing any software applications and in providing applications or software that is free-errors, user friendly and motivated. Thus, understanding the needs and requirements of human is necessary to gain benefits in enhancing the software design and in meeting people's expectation and experience individually and organizationally. Human factors need to be addressed in the early stage of any application and software design. Many software and application designers concern about the contents and load the human with noisy contents without fully paying attention to the flexibility of the design, the interaction and communication back and forth between human and computers. We will discuss issues that developers and designer should address during the development of any software or applications design. There are plenty of questions and issues which application and software designers need to take into account during the development of applications: who is the user? What does the user expect from pressing particular link or button? Does the user concern about the contents or the clarity of 
the content? What does the user expect from the design pattern that is used for common tasks? Does the user's expectation and experience meet across different user level? What color does the user like and dislike? What kind of data entry does the user like? Does the user like insertion or selection of data entries, etc?

The quick and short answer for the whole above issues is that there is a lack of conducting research on human factors and mis-fathom of what need to be done in the software design. In other words, there are several significant considerations: firstly, good understanding of the human behavior when interacting with Web application or software. Secondly, applying all the design technologies is beneficial to the advancement of traditional industries and the development of new businesses through creative collaborations, attractive Web application and software design between industries, human. And thirdly, consideration of the negative feedback from the human when interacting with the software systems or applications before launching the actual application or software system helps to resolve drawbacks of the system, enhances the design, and facilitates the interaction and design process as well.

We propose a Modified Prototype Model as our experimental research model. We use this model as it repeatedly involves the users in most of the stages of the Web application or software design and development. We use the user expertise in the stages of the design where necessary. It is used to explicate the positive and negative attitude toward coherent and legible designs. We are convinced that this model can be applied successfully in any application or software design and development. Human can give a positive feedback when they can fully enjoy the ease and the flexibility of the design, the legibility of the contents, and the ease of the navigation in any applications. The design of application or any software need to be studied carefully because any applications or software applications need to be delivered to the user with error-free, user friendly, coherent, and legible contents and structure, since the user interface is the essential component in communication between human and computers. Application and software companies need to carefully study the human capability in order to make it up to their standard and meet the their satisfaction, because individuals or users do not want to use application that has functional errors, full of unnecessary contents, incoherent structure, and unreadable contents. Moreover, designers need to make application more desirable, not too much colorful and full of media contents. In order to make an easy, usable, userfriendly and motivational application, first of all, the designers need to make forms more attractive and userfriendly with easy data entry which more into selection rather than typing, because designing interactive forms is really a big challenge. Second of all, designers need to provide easy navigation between in the application which user can easily recognize. Third of all, designers need to involve the user in the application development or design in order to achieve better user friendly applications.

\section{RELATED WORK}

Various researches have been ongoing to identify and to examine the human factors regarding to Web site and software application design. Many researchers have done some valuable research on how to make an interactive, and user-friendly Web site or software applications [6]. Lovett, et al. [7] conducted a research on studying the software design expenses which companies are paying for. In their research, they have not mentioned the methodology to study the human factors and how to design an interactive Web site that is easy to use and navigate through a Web page when a user interacting with a Web site. They have pointed that the lack of studying the importance of the usability. There are many companies which spend $\$ 2$ million per year on site or software redesign, without knowing if redesign made easier to use, because individuals do not meet the software or the Web application is user-friendly as they expected [7].

Salvedny and Smith [8] have studied the usability and the human factors in the automation design, they also have invested on the usability research and human factors in automation design and they have identified that usability is a necessary condition for survival. If a Web site is difficult to use, people leave. If the homepage fails to clearly state what a company offers and what users can do on the site, people leave. If users get lost on a Web site, they leave. If a Web site's information is hard to read or doesn't answer users' key questions, they leave. There is no such thing as a user reading a Web site manual or otherwise spending much time trying to figure out an interface. There are plenty of other Web sites available; leaving is the first line of defense when users encounter a difficulty. Therefore, it is worthy to invest on studying the human factor which can help to a better usability. More recently, a report of Hurts [9], founder of Creative Good, has meant to focus on online users and the result is that 39 percent of test shoppers have failed in their buying attempts because sites are too difficult to navigate. Additionally, 56 per cent of search attempts have been failed.

Basically, the above-mentioned researchers study and examine usability of the software design but they neglect the importance of the human factors, which mainly has significant impacts on the software quality. However, designing and building application that is user-friendly, readable and understandable from a designer perspective can be different from users' point of view. So we need to emphasize on the research to study and take human factors into consideration, for instance, culture, capabilities, language, etc. Utilizing and exploiting human needs, experience and requirements from different perspectives and desires can increase the curve of the 
profitability of the business strategy and enhance the application designs. Human factors principles have not been analyzed, utilized and internationalized individually or organizationally.

\section{MODIFIED PROTOTYPE MODEL}

We propose a modified prototyping model (MPM), which is a modified version of previous work. In comparison to previous work in $[10,11]$, they have not exploited and addressed users' feedback intensively like in our proposed model. Drawback of previous work may generally delay the developers at some steps of the design due to lack of users' feedbacks. Some of software engineers may wonder why we need to include the user feedback in every stage of the software design. It is simply because user needs system that is free-of-error and easy to interact. In order to do so, we need a user to feed us with opinions and comments to avoid any unwanted and undesirable features. The following steps explain the structure of our model in Figure 1:

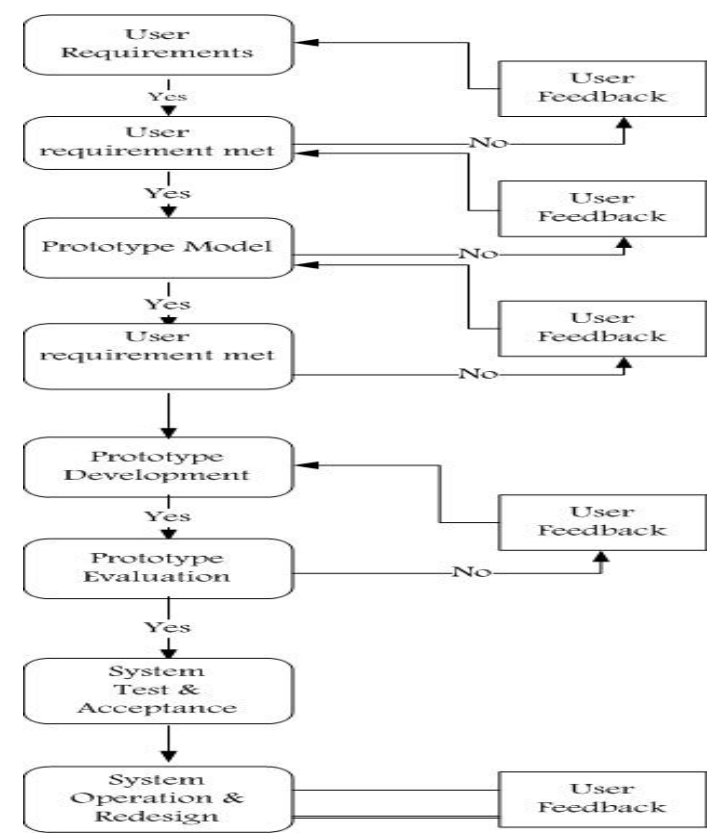

Fig.1. Structure of Modified Prototype Model

- Software designers (developers) need to gather all the user's requirements, and then, proceed to next step.

- If the user's requirements have been entirely met and the user was satisfied, then the developers need to proceed to next stage, else the developers need to go back to the previous stage and cover the missing things from the user's feedback.

- Iterative procedure repeats crossing the entire process of the design as in Figure 1.

\section{ANALYSIS METHODS}

In order to entirely capture and analyze the cognitive behavior of the individual while interacting with applications, we need special protocols and methods to capture the behavior of the individuals that can help us to predict what specifically the users expect from being interacting in the software applications, we have to find a way in which gives us the cognitive perception about the skills of the users from different aspects and perspectives. Understanding and identifying the cognitive and the physical behavior of individuals in the interaction will make it easier to design applications that are more motivational to individuals across different areas. Therefore, we propose analysis methods that assist to analyze and uncover human factors:

1) User feedback: involving the users in the early stage of any application or software design helps to uncover some of the drawbacks and unwanted features that individuals encounter while interacting with any applications.

2) Automated application agent: providing an automated agent in any application helps to discover the cognitive behavior and the impact of users' navigation. This agent should be able to extract some of the obstacles that human face during the interaction. Also, the agent must be able to correspond to the individuals' reaction and experience in the application.

3) Task analysis: analyzing the task that is provided in the application which users are appearing to be immersed. It can be determined by the number of times users clicks on a particular tasks.

4) User action: by analyzing the users' action, we can discover the user behavior or preferences. User action can help enhancing the application design. User action can be determined by the number of clicks and frequency of visits by identifying the users' IP addresses.

5) User interest: this method can explore users' interest by registering in the application and recommending it to others. Joining the application can tell that a particular user has been attracted to the application. However, leaving the application without leaving any feedback or joining is an unpleasant attitude toward the application from a user perspective. Therefore, we can say that the users are facing problems or are distracted either by finding the application is not interesting or by finding the application's content is not legible and has no coherent construction.

6) Online Survey: the purpose of online survey is to publish and distribute through internet to help uncovering the human capabilities and skill in the human computer interaction. It dramatically helps to discover the human factors globally and locally that provides the designer with overview of the human perception. 
7) Distributed Survey: is a type of questionnaire or other format that can be distributed among the users to uncover the users' experiences and abilities in human computer interaction. This type of survey can extremely assist in discovering what people like and dislike in the design.

8) User-centered design: designers should target the users. They should know who they are designing for, what users need, etc.

\section{EXPERIMENT}

For the experimental evaluation of the proposed research, we develop simple Web application that involves user feedback. We intend to involve the users in the requirements stage which meant to collect the user requirements including functional requirement and design requirements. (For the screenshots of the Web site, please visit http://mysite123.zxq.net/Website.html.) The purpose of the Web site is to serve users in online shopping, open discussion, download, and other features. We invite the users to help us clearly identify the requirements so that we can design according to the requirements. Overall, we receive many feedbacks which assisted us in making some modifications and eliminating some of the unnecessary contents. However, we receive a criticized feedback from the users. As we go through the design and development process further, we receive positive and negative feedbacks from users. Finally, we launch a Web site that is user-friendly, coherent structure, legible content, motivational to the user and meet users' expectations. As result, the users are pleased with a design and they find it motivated. Table 1 illustrates the users' opinions and perception about the Web site that we build and develop as our experimental research; it shows a varying percentage of users' responses in term of features and functionalities. The subjects of the experiments is students from one university in Pusan, they were graduate, and undergraduate students with different backgrounds. Our Website does not have a domain yet, so we use a local machine to build our experimental Web application.

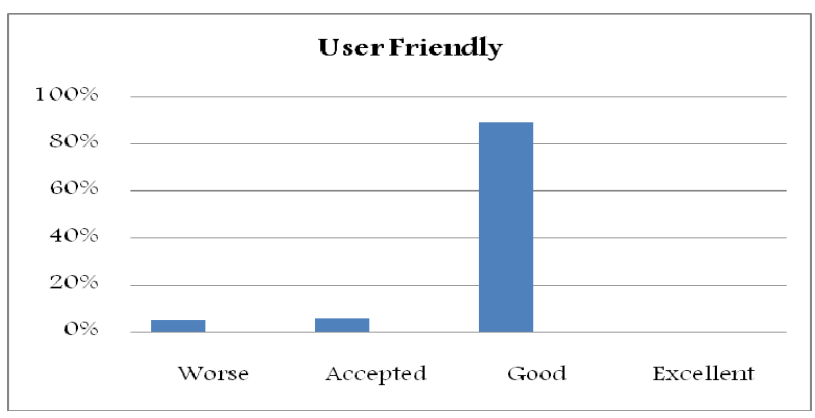

Fig.2. User's Responses
Fig. 2 shows the percentage of the user response for the Web application in term of user friendliness features. It shows that most of the candidates (89\%) respond that the Web application is 'user friendly' while the rest of the candidates $(6 \%)$ respond that it is 'acceptable', and $5 \%$ of the responses are marked as 'worse' in term of userfriendliness.

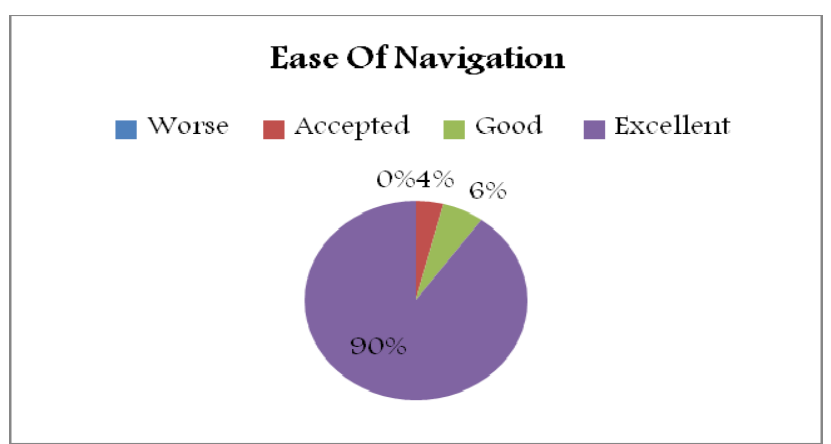

Fig. 3. User's Responses

Fig 3 displays the percentage of the users' response to ease of navigation feature. $90 \%$ of responders say it is 'excellent', 6\% responders say it is 'good', and 4\% responders say it is 'acceptable'.

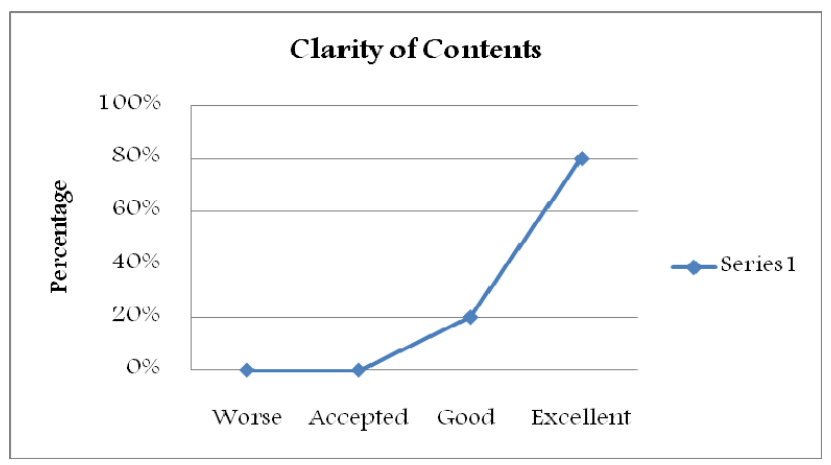

Fig. 4. User's Responses

Fig 4 shows also the variety of responses from the users interacting in the Web application. $80 \%$ of the users' responses are classed as 'excellent' and $20 \%$ of the users' responses are categorized as 'good' in term of clarity of contents.

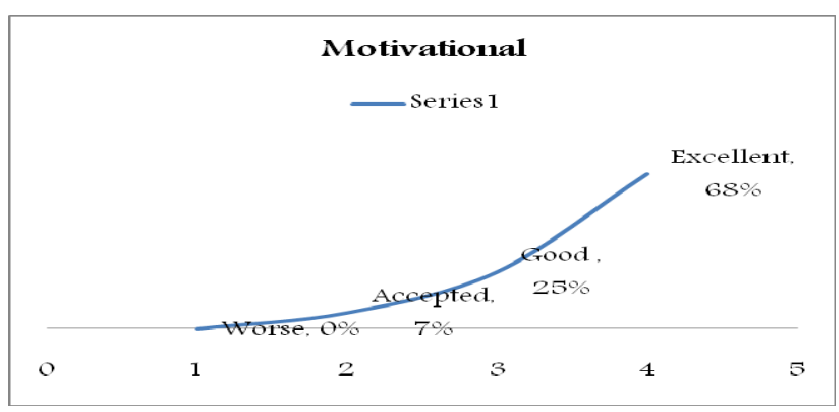

Fig. 5. User's Responses 
Fig 5 shows the responses of the various impression from the users while interacting in our experimental Web application that mainly focused to uncover human factors in software design. $68 \%$ of users response are denoted as 'excellent', $25 \%$ of users response are marked as 'good', and $7 \%$ of user response is 'acceptable' in term of motivational Web application.

\section{CONCLUSION}

Human factor is playing a significant role in software and applications design. Analyzing the human factors from different aspects and perspective helps to increase the software quality and demand. The purpose of this study is, to propose a model that can help designers and developers to uncover human factors that assist them to fulfill human requirements in term of user experience and usability. Designer needs to cover beyond the user expectation to make application more motivational and attractive. In addition, the main objective of studying the human factors in human computer interaction is to produce usable and safe systems to the users, as well as to construct functional systems.

\section{ACKNONWLEDGMENT}

This research was supported by the grant of Regional Innovation Center (RIC) program conducted by Korean Ministry of Knowledge Economy

\section{REFERENCES}

[1] Hewett, Baecker, Card, Carey, Gasen, Mantei, Perlman, Strong and Verplank, ACM SIGCHI Curricula for Human-Computer Interaction, USA. 2010

[2] Judith Crow, Computer Science, Laboratory SRI International Menlo Park CA 94025, USA, , Denis Javaux University of Li ège Work Psychology Dept FAPSE-ULG. Bat. B-32 4000 Sart -Tilman, elgium Design, Models and Mechanized Methods tha t Integrate Human Factors into Automation, 2002.

[3] Nigel Bevan.Usability Issues in Web Site Design, National Physic al Laboratory, Usability Services, Teddington, Middx, TW11 0 LW, UK, 2006.

[4] Robert J. K. Jacob and Keith S. Karn Eye Tracking in Hum an-Computer Interaction and Usability Research: Ready to De liver the Promises, 2008.

[5] Clark S. Turner, An Investigation of the Therac-25 Accidents, Nancy Leveson, University of Washington University of California, Irvine. 2003.

[6] Zhenhui Jiang, Jason Chan, Bernard C.Y. Tan, Wei Siong Ch ua, Effects of Interactivity on Website Involvement and Purch ase Intention.s, National University of Singapore, Singapore , 2010

[7] John Lovett with David Daniels, Lauren Jesuitus, Emily Murphy, Forrester, Web site spending trend, Shifting Budgets Amid Bullish Optimism.

[8] Gavriel Salvedny, Micheal J.Smith, Human Computer Interaction Software and Hardware Interfaces, Purdue University, West Lafayatte, USA.

[9] Mark Hurts, Joining strategy and usability. USA.2010.
[10] Gustav, Walter, Dieter, Prototyping-Oriented Software Development Concepts and Tools, Austria

[11] Nicky Nicky Danio, Human Computer Interaction and Your site, Nov 2002.

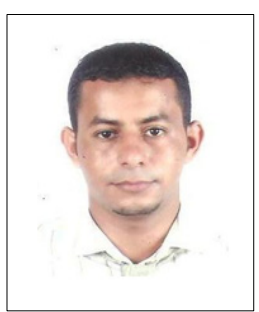

Sami Abduljalil is a Yemeni citizen who born on $1 / 2 / 1984$. He graduated as Bachelor Degree in Information System Egineering (Hons) Information technology in year 2010 at Multimedia Unversity (http://www.mmu.edu. my), Cyberjaya, Malaysia. He is currently also pursuing his master degree in Ubiquitous Computing at Dongseo University (http://www.dongseo.ac.kr).

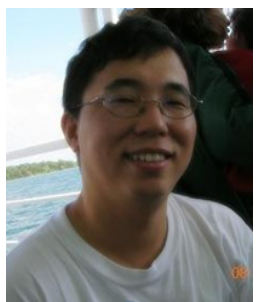

Dae-Ki Kang is an assistant professor at Dongseo University in South Korea. He was a senior member of engineering staff at the attached Institute of Electronics \& Telecommunications Research Institute in South Korea. He earned a $\mathrm{PhD}$ in computer science from Iowa State University in 2006. His research interests include social network services, machine learning, relational learning, statistical graphical models, metaheuristics, ontology learning, Tower of Hanoi, multimedia systems, intrusion detection, Web firewall, and computer vision. Prior to joining Iowa State, he worked at a Bay-area startup company and at Electronics and Telecommunication Research Institute in South Korea. He received a science master degree in computer science at Sogang University in 1994 and a bachelor of engineering (BE) degree in computer science and engineering at Hanyang University in 1992. 